\title{
Rational smoothness and affine Schubert varieties of type $A$
}

\author{
Sara Billey and Andrew Crites $\rrbracket$ \\ Department of Mathematics, University of Washington, Seattle, WA, USA
}

\begin{abstract}
The study of Schubert varieties in $G / B$ has led to numerous advances in algebraic combinatorics and algebraic geometry. These varieties are indexed by elements of the corresponding Weyl group, an affine Weyl group, or one of their parabolic quotients. Often times, the goal is to determine which of the algebraic and topological properties of the Schubert variety can be described in terms of the combinatorics of its corresponding Weyl group element. A celebrated example of this occurs when $G / B$ is of type $A$, due to Lakshmibai and Sandhya. They showed that the smooth Schubert varieties are precisely those indexed by permutations that avoid the patterns 3412 and 4231 . Our main result is a characterization of the rationally smooth Schubert varieties corresponding to affine permutations in terms of the patterns 4231 and 3412 and the twisted spiral permutations.

Résumé. L'étude des variétés de Schubert dans $G / B$ a mené à plusieurs avancées en combinatoire algébrique. Ces variétés sont indexées soit par l'élément du groupe de Weyl correspondant, soit par un groupe de Weyl affine, soit par un de leurs quotients paraboliques. Souvent, le but est de déterminer quelles propriétés algébriques et topologiques des variétés de Schubert peuvent être décrites en termes des propriétés combinatoires des éléments du groupe de Weyl correspondant. Un exemple bien connu, dû à Lakshmibai et Sandhya, concerne le cas où $G / B$ est de type $A$. Ils ont montré que les variétés de Schubert lisses sont exactement celles qui sont indexées par les permutations qui évitent les motifs 3412 et 4231 . Notre résultat principal est une caractérisation des variétés de Schubert lisses et rationnelles qui correspondent à des permutations affines pour les motifs 4231 et 3412 et les permutations spirales tordues.
\end{abstract}

Keywords: pattern avoidance, affine permutations, Schubert varieties

\section{Introduction}

The study of Schubert varieties and their singular loci incorporates tools from algebraic geometry, representation theory, and combinatorics. One celebrated result in this area due to Lakshmibai-Sandhya is that, in classical type $A$, the smooth Schubert varieties are precisely those that are indexed by permutations that avoid the patterns 4231 and 3412 [22], see also [29; 34]. A second important theorem in this area concerns a weaker notion than smoothness based on cohomology, called rational smoothness. Other generalizations of smoothness can be found in [32]. In general, smoothness implies rational smoothness, but not conversely. For classical Schubert varieties, Peterson showed smoothness is equivalent to rational

\footnotetext{
${ }^{\dagger}$ The authors acknowledge support from grant DMS-0800978 from the National Science Foundation. billey@uw. edu, acrites@uw.edu

1365-8050 @ 2011 Discrete Mathematics and Theoretical Computer Science (DMTCS), Nancy, France
} 
smoothness precisely in types $A, D, E$. Recently, there has been a surge of research activity related to affine Schubert varieties [2, 20, 21, 23, 24, 25, 27]. It is natural to ask how properties of smoothness, rational smoothness, singular loci and tangent spaces for affine Schubert varieties relate to their classical counterparts.

This paper summarizes the results of [1], which give a criterion for detecting rationally smooth Schubert varieties in affine type $A$. These varieties are indexed by the set of affine permutations, denoted $\widetilde{S}_{n}$. Generalizing the theorem of Lakshmibai-Sandhya, it was shown that the patterns 4231 and 3412 can be interpreted as patterns for affine permutations. A permutation avoiding these two patterns will again index a rationally smooth affine Schubert variety. However, there is an infinite family of affine permutations in $\widetilde{S}_{n}$ which contain 3412 and yet index rationally smooth affine Schubert varieties. These varieties are related to the spiral varieties studied by Mitchell [28], see also [2]. Thus, the main result is a complete characterization of the rationally smooth Schubert varieties in $G / B$ in type $\widetilde{A}_{n}$.

Theorem 1.1 [1, Theorem 1.1] Let $w \in \widetilde{S}_{n}$ for $n \geq 3$. The affine Schubert variety $X_{w}$ is rationally smooth if and only if one of the following hold:

1. $w$ avoids the patterns 3412 and 4231 ,

2. $w$ is a twisted spiral permutation (defined in Section 4.1).

Note, $\widetilde{S}_{2}$ is the infinite dihedral group. It follows that $X_{w}$ is rationally smooth for all $w \in \widetilde{S}_{2}$. Hence, throughout this paper we will assume $n \geq 3$ unless otherwise specified.

If a point $p \in X_{w}$ is not rationally smooth, then $p$ must be singular. Hence we get a necessary condition to detect singular affine Schubert varieties.

Corollary 1.2 Let $w \in \widetilde{S}_{n}$ for $n \geq 3$. If $w$ contains either a 3412 or 4231 pattern, then $X_{w}$ is singular.

The outline of this paper is as follows. In Section 2, we briefly introduce affine permutations. In Section 3 we then define the associated geometric objects, the affine Schubert varieties. Section 4 contains an outline of the proof of Theorem 1.1, and Section 5 discusses some corollaries of Theorem 1.1 and directions for future research.

\section{Affine permutations}

Let $\widetilde{S}_{n}$ be the group of bijections $w: \mathbb{Z} \rightarrow \mathbb{Z}$ such that the following two properties hold:

1. $w(i+n)=w(i)+n$ for all $i \in \mathbb{Z}$,

2. $\sum_{i=1}^{n} w(i)=\left(\begin{array}{c}n+1 \\ 2\end{array}\right)$.

Here the group operation is function composition. Elements of $\widetilde{S}_{n}$ are called affine permutations. Affine permutations first appeared in [26, §3.6] and were then further studied in [30]. See [6] for more background.

We can view an affine permutation in its one-line notation as the infinite string

$$
\cdots w_{-1} w_{0} w_{1} w_{2} \cdots w_{n} w_{n+1} \cdots
$$


where, for ease of notation, we write $w_{i}=w(i)$. An affine permutation is completely determined by its action on any window of $n$ consecutive indices $\left[w_{i}, w_{i+1}, \ldots, w_{i+n-1}\right]$. In particular, it is enough to record the base window $\left[w_{1}, \ldots, w_{n}\right]$ to capture all the information about $w$.

As a Coxeter group, $\widetilde{S}_{n}$ is generated by the set of simple reflections $S=\left\{s_{0}, s_{1}, \ldots, s_{n-1}\right\}$, where $s_{i}$ exchanges $i+k n$ and $i+1+k n$ for all $k \in \mathbb{Z}$ and fixes all other values. The relations amongst these generators are encoded in the following Coxeter graph.

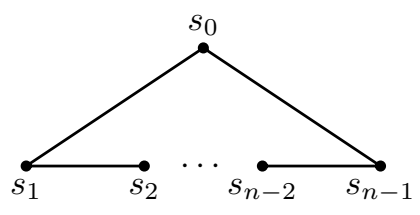

Fig. 1: Coxeter graph for $\widetilde{S}_{n}$.

As a group, $\widetilde{S}_{n}$ is also generated by the reflections $t_{i j}$ for $i<j$ and $i \not \equiv j(\bmod n)$. Here $t_{i j}$ interchanges $i+k n$ and $j+k n$ for all $k \in \mathbb{Z}$ and leaves all other values fixed. Since $t_{i j}=t_{i+n, j+n}$, it suffices to work with the set

$$
T=\left\{t_{i j}: 1 \leq i \leq n, i<j \text {, and } i \not \equiv j(\bmod n)\right\}
$$

Each $w \in \widetilde{S}_{n}$ can be written (in infinitely many ways) as a product of elements from the generating set $S$. We denote the minimal length of such an expression as $\ell(w)$, and call this statistic the length of $w$. An alternative description of this statistic (which is easier to compute) is given by the following proposition.

Proposition 2.1 [6, Proposition 8.3.1] Let $w \in \widetilde{S}_{n}$. Then

$$
\ell(w)=\#\left\{(i, j): 1 \leq i \leq n, i<j, \text { and } w_{i}>w_{j}\right\}
$$

There is a partial order on $\widetilde{S}_{n}$ defined as the transitive closure of the covering relations, where $u \lessdot v$ if there exists a reflection $t \in T$ with $u t=v$ and $\ell(u)=\ell(v)-1$. This partial order is called the Bruhat order on $\widetilde{S}_{n}$. Under Bruhat order, $\widetilde{S}_{n}$ is a ranked poset, ranked by the length function.

Given two elements $u \leq w$ related by Bruhat order, let $[u, w]=\left\{v \in \widetilde{S}_{n}: u \leq v \leq w\right\}$ denote the interval between $u$ and $w$. Call the interval [Id, $w]$ the order ideal of $w$, where $\mathrm{Id} \in \widetilde{\widetilde{S}}_{n}$ is the identity permutation. The Poincaré polynomial $P_{w}(q)$ is the rank generating function for the order ideal of $w$. Specifically,

$$
P_{w}(q)=\sum_{v \leq w} q^{\ell(v)} .
$$

This polynomial gets its name from the fact that $P_{w}\left(q^{2}\right)$ is the Poincare polynomial of the cohomology ring of $X_{w}$. Note that $v \leq w$ if and only if $v^{-1} \leq w^{-1}$, so we have $P_{w}(q)=P_{w^{-1}}(q)$.

We use the following theorem due to Carrell-Peterson to define rational smoothness of a Schubert variety in terms of the combinatorics of the Bruhat order and Coxeter groups. For $x, w \in \widetilde{S}_{n}$, let $\mathcal{R}(x, w)=\{t \in T: x<x t \leq w\}$. For any polynomial $f(q)$ of degree $n$, call $f$ palindromic if $f(q)=q^{n} f\left(q^{-1}\right)$. 
Theorem 2.2 [9. Theorem E] Let $W$ be an (affine) Weyl group (e.g., $W=\widetilde{S}_{n}$ ) and let $w \in W$. Then the following are equivalent.

1. The (affine) Schubert variety $X_{w}$ is rationally smooth.

2. $P_{w}(q)$ is palindromic.

3. $\# \mathcal{R}(x, w)=\ell(w)-\ell(x)$ for all $x \leq w$.

Our main result in Theorem 1.1 provides an efficient criterion for the three equivalent conditions in Theorem 2.2 in the case $W$ is the affine Weyl group of type $A$.

\section{Affine Schubert varieties}

We first briefly review the classical, non-affine case. Let $G$ be a connected, reductive algebraic group such as $G L_{n}(\mathbb{C})$. Let $B$ be a Borel subgroup of $G$. For example, if $G=G L_{n}(\mathbb{C})$, we can take $B$ to be the upper triangular matrices. The cosets $G / B$ form the points of a flag variety. For each $G$, there is an associated finite Weyl group $W$. For each $w \in W$, we obtain the Schubert variety $X_{w}$ by taking the Zariski closure of the orbit $B \cdot e_{w}$, where $e_{w}$ represents $w$ embedded in $G$. There are many good references on Schubert varieties including $[8,12 ; 13 ; 15 ; 16 ;, 17,33]$.

We now move to the construction of the affine Schubert varieties of type $A$. For an excellent reference on this construction, see [27] and the references contained therein. Let $G=\mathrm{GL}_{n}(\mathbb{C}((t)))_{0}$ be the group of invertible $n \times n$ matrices whose entries are rational functions in $t$ and whose determinant has order 0 . Each affine permutation corresponds to a matrix in $\widetilde{G}$ as follows. Let $w \in \widetilde{S}_{n}$ and for each $1 \leq i \leq n$, write $w_{i}=a_{i}+b_{i} n$, where $1 \leq a_{i} \leq n$. Then $\left\{a_{1}, \ldots, a_{n}\right\}=\{1, \ldots, n\}$ and $\sum_{i=1}^{n} b_{i}=0$ by the requirements 1 and 2 in the definition of an affine permutation. Set $e_{w}=\left(m_{i, j}\right)_{i, j=1}^{n}$, where $m_{i, a_{i}}=t^{b_{i}}$ and all other entries are 0 . Such a matrix is call an affine permutation matrix.

Example 3.1 Let $w=[8,-1,6,3,1,4] \in \widetilde{S}_{6}$. Then $\left(a_{1}, \ldots, a_{6}\right)=(2,5,6,3,1,4)$ and $\left(b_{1}, \ldots, b_{6}\right)=$ $(1,-1,0,0,0,0)$. Hence

$$
e_{w}=\left[\begin{array}{cccccc}
0 & t & 0 & 0 & 0 & 0 \\
0 & 0 & 0 & 0 & t^{-1} & 0 \\
0 & 0 & 0 & 0 & 0 & 1 \\
0 & 0 & 1 & 0 & 0 & 0 \\
1 & 0 & 0 & 0 & 0 & 0 \\
0 & 0 & 0 & 1 & 0 & 0
\end{array}\right] .
$$

The affine flag manifold is the space of cosets in $\widetilde{G} / \widetilde{B}$ where

$$
\widetilde{B}=\left\{b \in \mathrm{GL}_{n}(\mathbb{C}[[t]]):\left.b\right|_{t=0} \text { is upper triangular }\right\} .
$$

Note that the entries of elements in $\widetilde{B}$ are formal power series in $t$. A coset $g \widetilde{B}$ in $\widetilde{G} / \widetilde{B}$ has a unique representative that is in column echelon form. The pivot points in this echelon form will correspond to an affine permutation matrix $e_{w}$. The $\widetilde{B}$-orbit of this coset will then give all matrices in $\widetilde{G}$ whose echelon forms correspond to $e_{w}$. The set $C_{w}=\widetilde{B} e_{w} \widetilde{B} / \widetilde{B}$ is called the Schubert cell corresponding to $w$. 
Example 3.2 For $w=[8,-1,6,3,1,4], C_{w}$ is the set of all matrices whose column echelon form is

$$
\left[\begin{array}{cccccc}
a+b t & t & c & d & e t^{-1}+f & g \\
0 & 0 & 0 & 0 & t^{-1} & 0 \\
h & 0 & i & j & k & 1 \\
\ell & 0 & 1 & 0 & 0 & 0 \\
1 & 0 & 0 & 0 & 0 & 0 \\
0 & 0 & 0 & 1 & 0 & 0
\end{array}\right]
$$

Let $X_{w}=\bar{C}_{w}$ be the Zariski closure of the Schubert cell corresponding to $w$. We call $X_{w}$ the affine Schubert variety corresponding to $w$.

\section{Sketch of proof of Theorem 1.1}

\subsection{Factoring the Poincaré polynomial}

First suppose that $w \in \widetilde{S}_{n}$ avoids the patterns 3412 and 4231. By Theorem 2.2. we must show that $P_{w}(q)$ is palindromic. In particular, each $w$ with a palindromic Poincaré polynomial will factor using a parabolic decomposition as follows. Given a subset $J \subseteq S$ of the generators for a Coxeter group $W$, we can define $W_{J}$ to be the subgroup of $W$ generated by the elements of $J . W_{J}$ is called the parabolic subgroup generated by $J$. Each coset in $W / W_{J}$ contains a unique element of minimal length [18. Proposition 1.10]. The set of all minimal length coset representatives is denoted

$$
W^{J}=\{w \in W \mid \ell(w s)>\ell(w) \text { for all } s \in J\}
$$

and hence we can identify $W^{J}$ with these cosets. Similarly, let ${ }^{J} W$ denote the minimal length left coset representatives in $W_{J} \backslash W$. Bruhat order on $W$ induces partial orders on ${ }^{J} W$ and $W^{J}$. When we wish to refer to the Poincaré polynomial of a minimal length coset representative $w$ for the induced order on either of the quotients ${ }^{J} W$ or $W^{J}$, we will denote it by ${ }^{J} P_{w}$ or $P_{w}^{J}$, respectively. The parabolic decomposition for elements of $W$ is given as follows.

Proposition 4.1 [6, Proposition 2.4.4,2.5.1] For every $w \in W$ there exists a unique $u \in W_{J}$ and a unique $v \in{ }^{J} W$ such that $w=u \cdot v$ and $\ell(w)=\ell(u)+\ell(v)$. Moreover, the map $w \mapsto v$ is order preserving as a map from $W$ to the set of minimal length coset representatives.

Let $m(w, J)$ be the unique maximal element in $[0, w] \cap W_{J}$ (see [4, Theorem 2.2]). Computing $m(w, J)$ is equivalent to taking the Demazure product from [19. Definition 3.1] of the subword of $s_{i_{1}} \cdots s_{i_{p}}$ consisting of all of the letters from $J$. Then the following theorem shows how this parabolic decomposition gives rise to a factorization of the Poincaré polynomial.

Theorem 4.2 [3], Theorem 6.4] Suppose $w \in W$ has the parabolic decomposition $w=u \cdot v$ with $u \in W_{J}$ and $v \in{ }^{J} W$. If $u=m(w, J)$, then $P_{w}(q)=P_{u}(q) \cdot{ }^{J} P_{v}(q)$.

Returning to the case where $w$ avoids 3412 and 4231, we show that we can decompose $w$ as $w=u \cdot v$, where $u=m(w, J)$ for some $J \subset S$. Moreover, ${ }^{J} P_{v}(q)$ will be palindromic and $u$ avoids 3412 and 4231. We then use induction on $\ell(w)$. Thus, we have outlined the proof for the following theorem. 
Theorem 4.3 Let $w \in \widetilde{S}_{n}$ be an affine permutation that avoids the patterns 3412 and 4231 . Then $P_{w}(q)$ is palindromic.

Recall that, in the non-affine case, Gasharov shows that the factors of the Poincare polynomial for (rationally) smooth elements are all of the form $\left(1+q+\cdots+q^{k}\right)$ (see [14]). However, for $w \in \widetilde{S}_{n}$, these factors sometimes end up being the more general $q$-binomial coefficients instead.

\subsection{Twisted spiral permutations}

There is one important infinite family of affine permutations consisting of permutations that contain 3412 , but are still rationally smooth. For $a, b \in \mathbb{Z}$, define

$$
c(a, b)= \begin{cases}s_{a} s_{a+1} s_{a+2} \cdots s_{a+b-1}, & \text { if } b>0, \\ s_{a} s_{a-1} s_{a-2} \cdots s_{a+b+1}, & \text { if } b<0, \\ 1, & \text { if } b=0\end{cases}
$$

where all of the subscripts are taken modulo $n$. By definition, we have $c(a, 1)=c(a,-1)=s_{a}$, and $\ell(c(a, b))=|b|$. For any $k \in \mathbb{Z}$ with $k \neq 0$ and any $1 \leq i \leq n$, let

$$
w^{(i, k)}=w_{0}^{J_{i}} c(i, k(n-1)) \in \widetilde{S}_{n}
$$

where $w_{0}^{J_{i}}$ is the unique longest element in the parabolic subgroup generated by $J_{i}:=S \backslash\{i\}$. For $k \neq 0$, we call $w^{(i, k)}$ a twisted spiral permutation, since its reduced expression is obtained by spiraling around the Coxeter graph for $\widetilde{S}_{n}$ and then twisting by $w_{0}^{J_{i}}$.

By a result of Mitchell [28], the Poincaré polynomial for $c(i, k(n-1))$ in the quotient ${ }^{J_{i}}\left(\widetilde{S}_{n}\right)$ is palindromic (in fact, it is a $q$-binomial coefficient). Theorem 4.2 then shows that we may lift each $c(i, k(n-1)$ ) to a palindromic element in $\widetilde{S}_{n}$ by left multiplying by $w_{0}^{J_{i}}$. Hence the Poincare polynomial for $w^{(i, k)}$ is palindromic.

\subsection{When $w$ contains 4231 or 3412}

The converse to Theorem 1.1 asserts that the rationally smooth varieties found in Section 4.1 are the only ones that are rationally smooth. In the case where $w$ contains 4231, we show that the Poincaré polynomial fails to be palindromic at degree 1. The coefficient of $q$ in $P_{w}(q)$ is the number of distinct generators occurring in any reduced expression for $w$. The coefficient of $q^{\ell(w)-1}$ is the number of elements $v \leq w$ with $\ell(v)=\ell(w)-1$. Hence we construct a graph whose edges represent these covering relations and argue that there are more edges than distinct generators.

The case where $w$ contains 3412 and avoids 4231 is more complicated, in the sense that the Poincare polynomial can fail to be palindromic at various degrees. For this, we introduce an affine version of Bruhat pictures, which first appeared in [5]. We use these pictures to flatten a pair $x<w$ as much as possible, while preserving the length difference and the size of the set $\mathcal{R}(x, w)=\{t \in T: x<x t \leq w\}$.

Define the rank function for $w \in \widetilde{S}_{n}$ by

$$
r_{w}(p, q)=\#\left\{i \leq p: w_{i} \geq q\right\} .
$$

Define the difference function for the pair $x, w \in \widetilde{S}_{n}$ by

$$
d_{x, w}(p, q)=r_{w}(p, q)-r_{x}(p, q) .
$$


The difference function gives another useful characterization of Bruhat order and generalizes the Ehresmann criterion for Bruhat order [11], see also [12].

Theorem 4.4 [6, Theorem 8.3.7] For $x, w \in \widetilde{S}_{n}, x \leq w$ if and only if $d_{x, w}(p, q) \geq 0$ for all $p, q \in \mathbb{Z}$.

Given an affine permutation $w \in \widetilde{S}_{n}$, we can visualize $w$ as $\left\{\left(i, w_{i}\right): i \in \mathbb{Z}\right\} \subset \mathbb{Z}^{2}$. We think of each pair $\left(i, w_{i}\right)$ as a point in the plane drawn in Cartesian coordinates. Let $\operatorname{pt}_{w}(i)=\left(i, w_{i}\right)$. Furthermore, when comparing two affine permutations $x, w$ in Bruhat order using the rank difference function $d_{x, w}$ and Theorem 4.4, it is useful to visualize the nonzero entries of the function $d_{x, w}$ as a union of shaded rectangles in the plane. Combining both visualizations we get an affine Bruhat picture.

For example, take $w=[8,3,1,0,4,5]$ and $x=w t_{1,4}=[0,3,1,8,4,5]$, then $d_{x, w}$ will be positive in the translated shaded regions of the affine Bruhat picture for $x<w$ in Figure 2. Here the points of $w$ are represented by dots, while the points of $x$ are represented by x's.

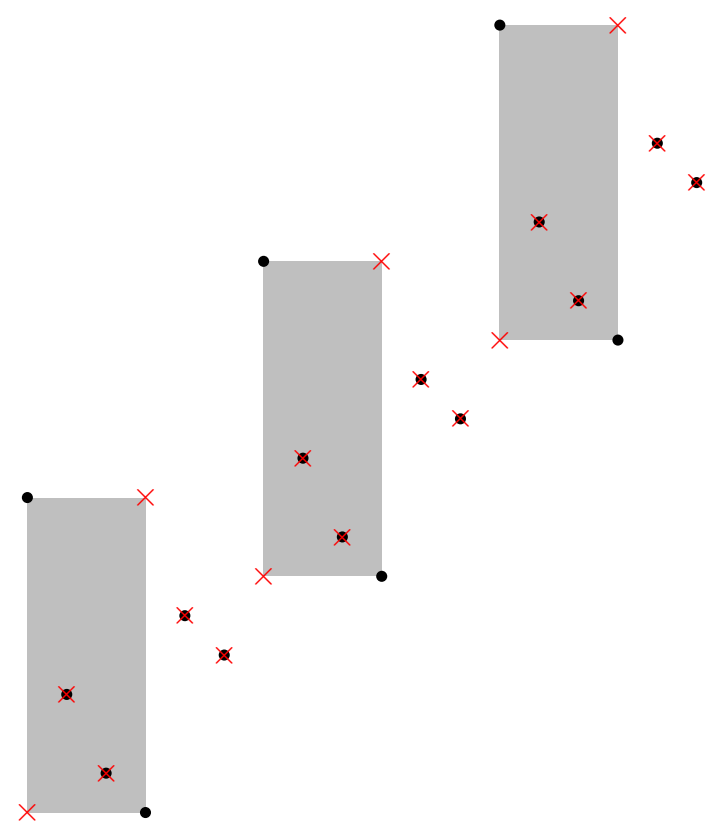

Fig. 2: $w=[8,3,1,0,5,4]$ and $x=w t_{1,4}=[0,3,1,8,5,4]$.

Given integers $p<q$ such that $x_{p}<x_{q}$, we will use the affine Bruhat pictures to determine if $x<$ $x t_{p, q} \leq w$. Observe that $d_{x, x t_{p, q}}$ is positive on the periodic union of rectangles

$$
\mathcal{A}_{p, q, k}(x)=[p+k n, q-1+k n] \times\left[x_{p}+1+k n, x_{q}+k n\right] \text {, for all } k \in \mathbb{Z} .
$$

It is possible that these rectangles overlap in affine Bruhat pictures as in Figure 3 . If a point $(i, j)$ is contained in exactly $m$ consecutive translates of $\mathcal{A}_{p, q, 0}(x)$, then $d_{x, x t_{p, q}}=m$. Thus, we get the following criterion for determining if $t_{p, q} \in \mathcal{R}(x, w)$. 


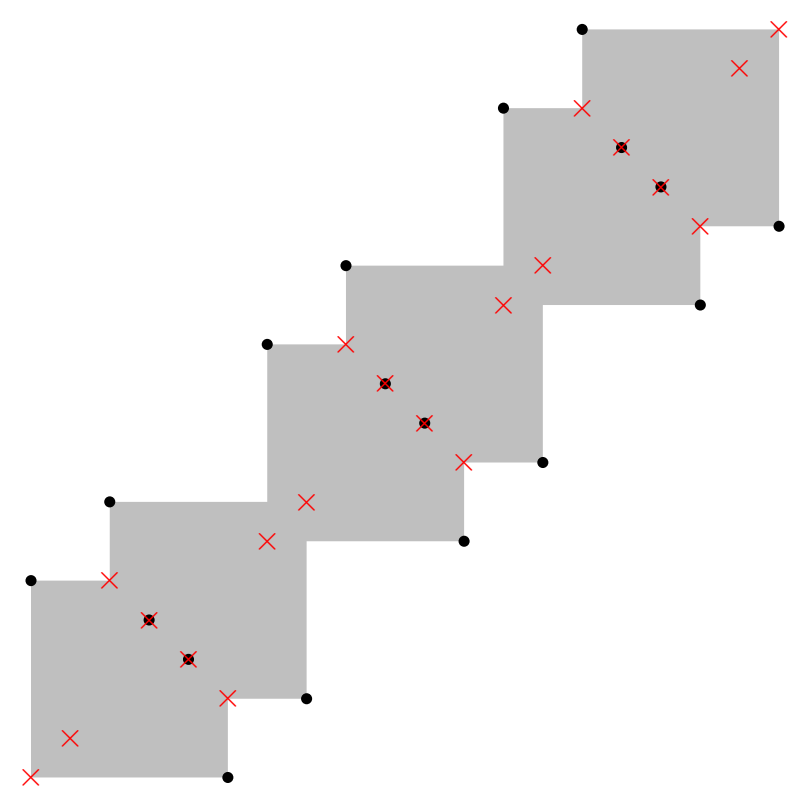

Fig. 3: $w=[6,-3,8,5,4,1]$ and $x=[1,2,6,5,4,3]$.

Lemma 4.5 Given affine permutations $x<w$ and integers $p<q$ such that $x_{p}<x_{q}$, then $t_{p, q} \in \mathcal{R}(x, w)$, provided $d_{x, w}(i, j) \geq m$ for every $(i, j)$ contained in $m$ consecutive rectangles $\mathcal{A}_{p, q, k}(x)$.

As an example of computing $\mathcal{R}(x, w)$ using shading, let $w=[6,-3,8,5,4,1]$ and $x=[1,2,6,5,4,3]$ (see Figure 3). Then

$$
\mathcal{R}(x, w)=\left\{t_{12}, t_{13}, t_{14}, t_{15}, t_{16}, t_{23}, t_{24}, t_{25}, t_{26}, t_{37}, t_{38}, t_{47}, t_{48}, t_{57}, t_{58}, t_{67}, t_{68}\right\} .
$$

If $w$ contains 3412 but avoids 4231, and $w$ is not a twisted spiral, we identify a specific affine permutation $x<w$ such that $\# \mathcal{R}(x, w)>\ell(\omega)$ by using the Bruhat pictures. Then, we can appeal to Theorem 2.2 The proof requires analyzing several cases which whittle down the possible Bruhat pictures until only the twisted spirals remain. As mentioned about however, the twisted spirals are rationally smooth. This completes the outline of the proof of the following theorem.

Theorem 4.6 If $w \in \widetilde{S}_{n}$ contains either a 3412 or a 4231 pattern, but is not a twisted spiral permutation, then $P_{w}(q)$ is not palindromic.

\section{Further directions}

It was shown in [10] that there are only a finite number of 3412 avoiding affine permutations in $\widetilde{S}_{n}$. Thus, there exists a finite number of smooth Schubert varieties indexed by $\widetilde{S}_{n}$. Using this fact, we have verified the following conjecture up to $n=5$. Significant progress on this conjecture has since been made by Cheng, Crites, and Kuttler, the results of which are currently in preparation. 
Conjecture 1 Let $w \in \widetilde{S}_{n}$. The Schubert variety $X_{w}$ is smooth if and only if $w$ avoids 3412 and 4231.

In fact, we can get a tighter bound on the length of any $w$ avoiding 3412 and 4231 . In the proof of Theorem 1.1, we proved that any 3412 and 4231 avoiding affine permutation can be written in the form $w=w^{\prime} \sigma$, with $\ell(w)=\ell\left(w^{\prime}\right)+\ell(\sigma)$. Both $w^{\prime}$ and $\sigma$ are elements of a proper parabolic subgroup of $\widetilde{S}_{n}$, and hence $\ell\left(w^{\prime}\right), \ell(\sigma) \leq\left(\begin{array}{l}n \\ 2\end{array}\right)$. Thus, we have the following corollary.

Corollary 5.1 (To proof of Theorem 1.1) If $w \in \widetilde{S}_{n}$ avoids 3412 and 4231, then $\ell(w) \leq 2\left(\begin{array}{c}n \\ 2\end{array}\right)$.

Since the number of affine permutations of length at most $2\left(\begin{array}{c}n \\ 2\end{array}\right)$ is finite, we would like to compute how many affine permutations in $\widetilde{S}_{n}$ avoid both 3412 and 4231 . Conjecturally, this is equivalent to the number of smooth affine Schubert varieties of type $\widetilde{A}_{n}$. In general, not much is known about the number of affine permutations that avoid a given set of patterns, except for a characterization of when this number is finite [10]. Starting with $n=2$, the first few terms of this sequence are $5,31,173,891,4373$, which now appear in Sloane's [31] as sequence A180635.

In [7], Björner and Ekedahl give general inequalities amongst the coefficients of the Poincaré polynomial for elements of any crystallographic Coxeter group. As mentioned in Section 4.3, we showed that when $w$ contains 4231 , the coefficient of $q$ in $P_{w}(q)$ is strictly less than the coefficient of $q^{\ell(w)-1}$. Combining this fact with [7, Theorem C], proves the following corollary.

Corollary 5.2 Let $w \in \widetilde{S}_{n}$ and assume $w$ contains a 4231. Then if $P_{\mathrm{Id}, w}=1+a_{1} q+\cdots+a_{d} q^{d}$ is the Kazhdan-Lusztig polynomial indexed by Id, w, then $a_{1}>0$.

\section{References}

[1] S. Billey AND A. CRITES, Pattern characterization of rationally smooth Schubert varieties of type A. preprint, math.CO/1008.5370v1, August 2010.

[2] S. Billey And S. A. Mitchell, Affine partitions and affine Grassmannians, Electron. J. Combin., 16 (2009), pp. Research Paper 18, 45 pp. (electronic).

[3] S. Billey And A. PostniKov, Smoothness of Schubert varieties via patterns in root subsystems, Adv. in Appl. Math., 34 (2005), pp. 447-466.

[4] S. C. Billey, C. K. FAn, And J. Losonczy, The parabolic map, J. Algebra, 214 (1999), pp. 1-7.

[5] S. C. Billey And G. S. Warrington, Maximal singular loci of Schubert varieties in $\operatorname{SL}(n) / B$, Trans. Amer. Math. Soc., 355 (2003), pp. 3915-3945 (electronic).

[6] A. BJÖRner And F. BREnTI, Combinatorics of Coxeter groups, vol. 231 of Graduate Texts in Mathematics, Springer, New York, 2005.

[7] A. BJÖRNER AND T. EKEDAHL, On the shape of Bruhat intervals, Ann. of Math. (2), 170 (2009), pp. 799-817.

[8] M. BRION, Lectures on the geometry of flag varieties, in Topics in cohomological studies of algebraic varieties, Trends Math., Birkhäuser, Basel, 2005, pp. 33-85. 
[9] J. B. CARRELL, The Bruhat graph of a Coxeter group, a conjecture of Deodhar, and rational smoothness of Schubert varieties, in Algebraic groups and their generalizations: classical methods (University Park, PA, 1991), vol. 56 of Proc. Sympos. Pure Math., Amer. Math. Soc., Providence, RI, 1994, pp. 53-61.

[10] A. CRITES, Enumerating pattern avoidance for affine permutations, Electron. J. Combin., 17 (2010), pp. Research Paper 127, 13 pp. (electronic).

[11] C. Ehresmann, Sur la topologie de certains espaces homogènes, Ann. of Math. (2), 35 (1934), pp. 396-443.

[12] W. Fulton, Young tableaux, vol. 35 of London Mathematical Society Student Texts, Cambridge University Press, Cambridge, 1997. With applications to representation theory and geometry.

[13] W. Fulton AND J. HarRis, Representation Theory, vol. 129 of Graduate Texts in Mathematics, Springer-Verlag, New York, 1991. A first course, Readings in Mathematics.

[14] V. Gasharov, Factoring the Poincaré polynomials for the Bruhat order on $S_{n}$, J. Combin. Theory Ser. A, 83 (1998), pp. 159-164.

[15] N. Gonciulea And V.Lakshmibai, Flag varieties, Hermann-Acutalities Mathematiques, 2001.

[16] J. HARRIS, Algebraic geometry, vol. 133 of Graduate Texts in Mathematics, Springer-Verlag, New York, 1995. A first course, Corrected reprint of the 1992 original.

[17] J. E. Humphreys, Linear algebraic groups, Springer-Verlag, New York, 1975. Graduate Texts in Mathematics, No. 21.

[18] _ Reflection groups and Coxeter groups, vol. 29 of Cambridge Studies in Advanced Mathematics, Cambridge University Press, Cambridge, 1990.

[19] A. Knutson And E. Miller, Subword complexes in Coxeter groups, Adv. Math., 184 (2004), pp. 161-176.

[20] V. Kreiman and V. LaKshmibai, Contributions to automorphic forms, geometry, and number theory, Johns Hopkins Univ. Press, 2004, ch. Richardson varieties in the Grassmannian, pp. 573597.

[21] J. Kuttler And V. Lakshmibai, Singularities of affine Schubert varieties, SIGMA Symmetry Integrability Geom. Methods Appl., 5 (2009), pp. Paper 048, 31.

[22] V. Lakshmibai And B. Sandhya, Criterion for smoothness of Schubert varieties in $\mathrm{SL}(n) / B$, Proc. Indian Acad. Sci. Math. Sci., 100 (1990), pp. 45-52.

[23] T. LAM, Affine Stanley symmetric functions, Amer. J. Math., 128 (2006), pp. 1553-1586.

[24] T. Lam, L. Lapointe, J. Morse, and M. Shimozono, Affine insertion and Pieri rules for the affine Grassmanian. To appear in Memoirs of the AMS. 
[25] T. LAM, A. SCHILling, AND M. SHIMOZONO, Schubert polynomials for the affine Grassmannian of the symplectic group, Math. Z., 264 (2010), pp. 765-811.

[26] G. LuszTig, Some examples of square integrable representations of semisimple p-adic groups, Trans. Amer. Math. Soc., 277 (1983), pp. 623-653.

[27] P. MagYaR, Affine Schubert varieties and circular complexes. preprint, math.AG/0210151v1, October 2002.

[28] S. A. Mitchell, A filtration of the loops on $\mathrm{SU}(n)$ by Schubert varieties, Math. Z., 193 (1986), pp. 347-362.

[29] K. M. Ryan, On schubert varieties in the flag manifold of $\operatorname{sl}(n, \mathbb{C})$, Math. Ann., (1987).

[30] J. Y. SHI, The Kazhdan-Lusztig cells in certain affine Weyl groups, vol. 1179 of Lecture Notes in Mathematics, Springer-Verlag, Berlin, 1986.

[31] N. J. A. SLOANE, Online encyclopedia of integer sequences. http://www.research.att. $\mathrm{com} / \sim \mathrm{njas} /$ sequences/, 2009.

[32] H. Ulfarsson, A unification of permutation patterns related to Schubert varieties. preprint, math.CO/1002.4361v4, January 2011.

[33] Wikipedia, Generalized flag variety - wikipedia, the free encyclopedia. http://en. wikipedia.org/wiki/Generalized_flag_variety, 2010. [Online; accessed 14-June2010].

[34] J. S. WOLPER, A combinatorial approach to the singularities of Schubert varieties, Adv. Math, 76 (1989), pp. 184-193. 
